\title{
Enhancing the Energy Efficiency of Fully Electric Vehicles via the Minimization of Motor Power Losses
}

\author{
A. Pennycott ${ }^{1}$, L. De Novellis ${ }^{1}$, P. Gruber ${ }^{1}$, A. Sorniotti ${ }^{1}$ and T. Goggia ${ }^{1,2}$ \\ ${ }^{1}$ Dept. of Mechanical Engineering Sciences \\ University of Surrey \\ Guildford, United Kingdom \\ a.pennycott@surrey.ac.uk \\ ${ }^{2}$ Dept. of Computer Engineering and Systems Science \\ University of Pavia \\ Pavia, Italy
}

\begin{abstract}
Individually-controlled powertrains of fully electric vehicles present an opportunity to enhance the steady-state and transient cornering response of a car via continuously-acting controllers and enable various "driving modes" to be available. This study investigates the associated potential for energy savings through the minimization of power losses from the motor units via wheel torque allocation. Power losses in straight-ahead driving and a ramp steer maneuver for different motor types and under different wheel torque allocation schemes are analyzed in an offline simulation approach. Significant reductions in motor power losses are achieved for two motor types using an optimization scheme based on look-up tables of motor loss data. Energy loss minimization cannot be achieved through a direct quadratic approximation of the power losses.
\end{abstract}

Keywords - vehicle dynamics; optimization; efficiency; control allocation

\section{INTRODUCTION}

Current vehicle stability control systems are primarily developed to enhance vehicle behavior under emergency conditions through temporary actuation of individual wheel brakes [1]. For fully electric vehicles equipped with individually-controlled powertrains, the control of vehicle behavior can be extended to all possible steady-state and transient driving conditions. For example, by developing continuously operating control systems, key vehicle properties such as the understeer characteristic (the standard graph used to describe the steady-state response of a vehicle to changes in the steering wheel angle) can be modified to achieve specified handling qualities [2]. As a result, different "driving modes" can be made available to the driver. For instance, "eco" driving modes, which emphasize energy efficiency, or "sport" driving modes, which may make the vehicle react aggressively to steering wheel inputs, are possible merely through changes in the control parameters.

Vehicles with four electric motors provide the greatest potential with respect to controlling handling and energy efficiency qualities because the configuration yields an overactuated system: targets concerning the net traction force and yaw moment can be met using an infinite number of combinations of the four wheel torques. This opens up a control allocation opportunity in which a secondary objective can be devised in addition to meeting the primary objective realizing the target traction force and yaw moment. Different methods of control allocation have been proposed which focus on criteria relating to tire forces, wheel slip [3-5] and motor efficiency [6,7]. Similarly, one investigation developed an offline optimization procedure to efficiently distribute torque and select the transmission state in a dual motor electric drivetrain [8]. Typically, the control allocation methods are based on minimizing a low order function such as a quadratic cost function [9], or alternatively, can be based on a set of rules [10].

There are various studies on the minimization of energy consumption through optimization-based control allocation $[6,7]$. These have mainly focused on potential energy savings for a specific type of motor that may not reflect the typical characteristics of the latest motor technology used in modern electric vehicles. As a result, the general applicability of the presented control allocation methods for diverse motor types remains unclear. Moreover, various options of varying complexity are available as cost functions for optimization, and the relative merits of these have not, to date, been evaluated.

In this paper, the potential for energy savings through minimizing motor power losses during control allocation is investigated through an offline simulation approach. To examine the influence of different motor characteristics, results are compared for three electric motor types. Furthermore, optimization schemes based on minimizing a direct quadratic cost function of the motor power loss are contrasted with schemes using look-up tables to determine the motor power losses at different motor speeds and torques. The comparisons are presented for two maneuvers types: straight-ahead driving, and a ramp steer maneuver, both at constant longitudinal acceleration. 


\section{METHODS}

\section{A. Overall Control Structure}

A simplified schematic of the overall control structure considered in the presented simulation study is shown in Figure 1. Based on the vehicle speed $(V)$, and acceleration $\left(u_{a c c}\right)$ and brake pedal $\left(u_{b r}\right)$ inputs, the drivability controller generates a demand for the net traction force $\left(F_{x}^{*}\right)$. The reference yaw rate controller produces a target yaw rate $\left(r^{*}\right)$ based on the steering wheel angle $(\delta)$, vehicle velocity, longitudinal acceleration $\left(a_{x}\right)$, friction coefficient $(\mu)$ and such that a target understeer characteristic is produced. The target yaw rate is determined from a look-up table which, in turn, is generated through an offline procedure detailed in [11]. The high level controller consists of a feedforward element and a proportional-integral-derivative feedback controller, and drives the actual yaw rate $(r)$ to the reference yaw rate through adjustment of the corrective yaw moment $\left(M_{z}^{*}\right)$.

The torque allocation block determines the wheel torque values at the four wheels of the car which meet the targets concerning the traction force and yaw moment $\left(F_{x}^{*}\right.$ and $\left.M_{z}^{*}\right)$. Lastly, the motor torques $\left(\boldsymbol{\tau}_{m}\right)$ and brake pressures $\left(\boldsymbol{p}_{b}\right)$ required to realize the desired wheel torques are calculated.

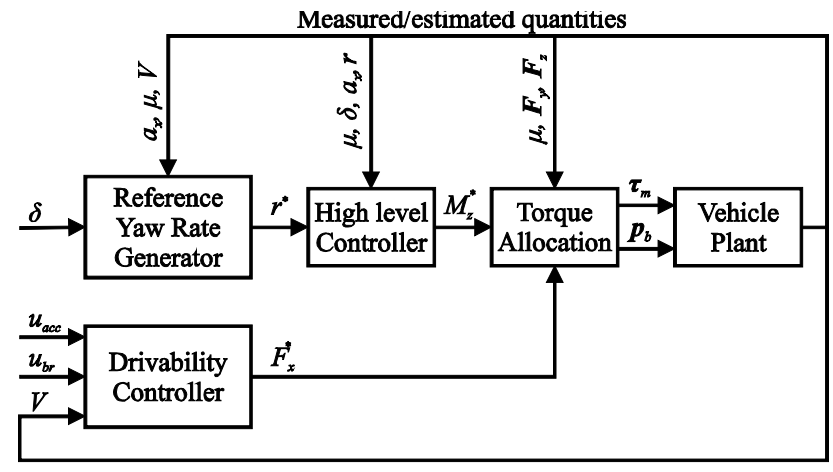

Figure 1. Overall control structure.

Hence, by controlling the individual wheel torques, the control system directly alters the cornering behavior, allowing various driving modes to be produced which can then be selected by the driver. For instance, with a "sport" driving mode, the high level controller determines the wheel torques that yield a low understeer gradient (so that the car is very responsive to changes in steering wheel angle), and also extends the understeer curve to realize greater maximum lateral acceleration levels. With an "eco" driving mode, energy efficiency can be given priority; the potential of this concept is examined here. In particular, an offline optimization procedure is used to assess the potential for energy savings of a motor power loss minimization scheme in the torque allocation block of Figure 1. The sections below detail how a cost function reflecting the motor power loss is formulated, and how the wheel torques are allocated based on the traction demand, yaw moment target and the motor power cost function.

To investigate the effectiveness of the wheel torque allocation utilizing the power loss minimization concept, results are compared with simulations using a simpler torque allocation method in which the wheel torques of the front and rear axles contribute equally to the traction and yaw moment demands.

\section{B. Control Allocation}

1) Relating the Traction Force and Yaw Moment with the Wheel Torques

The basic vehicle parameters half-track $\left(t_{h}\right)$ and wheel radius $\left(r_{w}\right)$ are used to relate the wheel torques at the four wheels (contained in vector $\boldsymbol{\tau}_{w}$ ) to the net traction force and yaw moment acting on the car. The basic vehicle geometry is shown in Figure 2. The subscripts 1 to 4 refer to variables of the front-left, front-right, rear-left and rear-right wheels, respectively, throughout this paper.

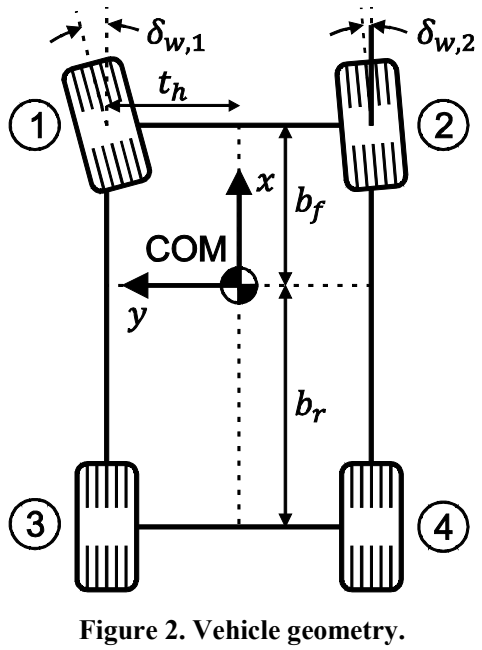

Using these basic parameters and, as a first approach, neglecting the steering angles of the front wheels $\left(\delta_{w, 1}\right.$ and $\left.\delta_{w, 2}\right)$, the net longitudinal force and yaw moment are

$$
\begin{gathered}
F_{x}=\frac{1}{r_{w}}\left(\tau_{w, 1}+\tau_{w, 2}+\tau_{w, 3}+\tau_{w, 4}\right) \\
M_{z}=\frac{1}{r_{w}}\left(-t_{h} \tau_{w, 1}+t_{h} \tau_{w, 2}-t_{h} \tau_{w, 3}+t_{h} \tau_{w, 4}\right) .
\end{gathered}
$$

Equation (2) does not consider the interaction between longitudinal and lateral tire forces, which is a secondary effect in most conditions.

\section{2) Evenly Distributed Control Allocation}

Under this scheme, the contributions from the wheels at the front and rear axles to the total longitudinal force are to be equal. Hence, the individual wheel torques must satisfy

$$
\frac{\tau_{w, 1}+\tau_{w, 2}}{\tau_{w, 1}+\tau_{w, 2}+\tau_{w, 3}+\tau_{w, 4}}=0.5
$$

Similarly, the front and rear axles are to produce equal contributions to the yaw moment, giving the equation

$$
\frac{-t_{h} \tau_{w, 1}+t_{h} \tau_{w, 2}}{-t_{h} \tau_{w, 1}+t_{h} \tau_{w, 2}-t_{h} \tau_{w, 3}+t_{h} \tau_{w, 4}}=0.5
$$


There are thus four linear equations (equations (1) - (4)) in the four wheel torques which allow the latter to be determined through matrix inversion. The solution obtained using this approach, referred to as $\boldsymbol{\tau}_{w}^{\text {even }}$, is

$$
\boldsymbol{\tau}_{w}^{\text {even }}=r_{w}\left[\begin{array}{l}
\frac{F_{x}}{4}-\frac{M_{z}}{4 t_{h}} \\
\frac{F_{x}}{4}+\frac{M_{z}}{4 t_{h}} \\
\frac{F_{x}}{4}-\frac{M_{z}}{4 t_{h}} \\
\frac{F_{x}}{4}+\frac{M_{z}}{4 t_{h}}
\end{array}\right]=\left[\begin{array}{l}
\tau_{w, l}^{\text {even }} \\
\tau_{w, r}^{\text {even }} \\
\tau_{w, l}^{\text {even }} \\
\tau_{w, r}^{\text {even }}
\end{array}\right]
$$

\section{3) Optimization Control Allocation}

The optimization scheme aims to produce a vector of wheel torques, $\boldsymbol{\tau}_{w}^{o p t i}$, that minimizes the total power loss from the motor drives and also meets the traction and yaw moment targets.

a) Control Effectiveness and Virtual Control: The targets for traction and yaw moment are contained within the virtual control vector, $\boldsymbol{v}=\left[\begin{array}{ll}F_{x}^{*} & M_{z}^{*}\end{array}\right]^{T}$. The control effectiveness matrix, $\boldsymbol{B}$, approximately relates the wheel torques to the actual traction and yaw moment according to the approximated equation

$$
\left[\begin{array}{c}
F_{x} \\
M_{z}
\end{array}\right] \approx \boldsymbol{B} \boldsymbol{\tau}_{w}
$$

Through inspection of equations (1) and (2),

$$
\boldsymbol{B}=\frac{1}{r_{w}}\left[\begin{array}{cccc}
1 & 1 & 1 & 1 \\
-t_{h} & t_{h} & -t_{h} & t_{h}
\end{array}\right]
$$

b) Optimization Constraints: If the virtual control is satisfied, then $\boldsymbol{v}=\boldsymbol{B} \tau_{w}$. In order to ensure that the virtual control is met, this equation is imposed as an equality constraint during optimization. In addition, there are minimum and maximum torque limits for each motor/generator unit, $i$. These depend on the motor speed and are thus of the form $\tau_{w, i}^{\min }=f_{\min }\left(\omega_{m, i}\right)$ and $\tau_{w, i}^{\max }=f_{\max }\left(\omega_{m, i}\right)$.

c) Motor Power Loss Cost Function: In order to produce a vector of four wheel torques that minimizes the total motor power loss, the latter must be represented as a function of the four wheel torques. A map (obtained empirically) is used to determine the efficiency of motor $i$ at different values of motor speed $\left(\omega_{m, i}\right)$ and motor torque $\left(\tau_{m, i}\right)$. The motor power loss at a certain motor speed and torque can be determined directly from this efficiency. Moreover, for a fixed transmission ratio ( $\kappa$, most of the electric drivetrains have a single-speed transmission) and neglecting the transmission efficiency, the motor torques are, in steady-state, related to the wheel torques via the relationship $\tau_{w, i}=\kappa \tau_{m, i}$.

The cost function must approximate the power loss of motor $i, P_{\text {loss }, i}$, at different locations in the speed-torque space as determined by the efficiency map. Two forms of power loss approximation are investigated in this work. Since many studies use a quadratic programming optimization approach, the first is a direct quadratic representation of the motor power loss.

$$
P_{l o s s, i}=\sum_{j=0}^{2} a_{j, i}\left(\omega_{m, i}\right) \tau_{w, i}^{2-j}
$$

For discrete levels of motor speed, the cost function coefficients $a_{j, i}$ are determined via a least squares minimization approach using the motor map data (described in section C.3). During wheel torque allocation, values of the cost function coefficients at arbitrary motor speeds are determined through interpolation.

The second approach uses the available motor efficiency data to generate a look-up table of motor loss data to be used in the optimization procedure. Given the motor power output, $P_{i}$, which is the product of $\tau_{m, i}$ and $\omega_{m, i}$, the corresponding power loss is then given by

$$
P_{\text {loss }, i}= \begin{cases}\left|P_{i}\right|\left(1 / \eta_{i}-1\right) & \text { if } \tau_{w, i} \geq 0 \\ \left|P_{i}\right|\left(1-\eta_{i}\right) & \text { if } \tau_{w, i}<0\end{cases}
$$

For both approaches to calculating $P_{l o s s, i}$, the total motor power loss across the four actuators is

$$
P_{\text {loss }}=\sum_{i=1}^{4} P_{\text {loss }, i}
$$

d) Optimization Solution: The optimization problem is solved using the MATLAB function fmincon, with the interior point method selected as the solution algorithm. A number of initial points are generated by varying the proportions of traction and yaw moment to be generated by the front wheels between 0.5 and 1 . Using these initial points, a number of local minima are determined and the local minimum with the lowest objective function is then selected.

e) Limitations of Quadratic Programming: The potential of using wheel torque allocation based on quadratic programming to minimize the total motor power loss is limited. This is demonstrated in the following analysis and also through the simulation approach of this investigation.

One possible solution satisfying the virtual control is $\boldsymbol{\tau}_{w}^{\text {even }}$, provided in equation (5). The general solution for the wheel torque vector $\left(\boldsymbol{\tau}_{w}\right)$ can be expressed in terms of two independent variables, $\Delta \tau_{w, l}$ and $\Delta \tau_{w, r}$.

$$
\boldsymbol{\tau}_{w}=\boldsymbol{\tau}_{w}^{\text {even }}+\left[\begin{array}{cc}
-1 & 0 \\
0 & -1 \\
1 & 0 \\
0 & 1
\end{array}\right]\left[\begin{array}{l}
\Delta \tau_{w, l} \\
\Delta \tau_{w, r}
\end{array}\right]
$$

In both straight-ahead driving and cornering maneuvers, the angular velocities of the wheels on the left-hand side of the vehicle are approximately equal, as are the wheels on the right-hand side. Therefore, the motor losses at the front-left and the rear-left wheels can be modeled using the same quadratic coefficients, with analogous coefficients for the right-hand side motors.

$$
\begin{aligned}
& P_{\text {loss }, 1 / 3}=a_{2, l} \tau_{w, 1 / 3}^{2}+a_{1, l} \tau_{w, 1 / 3}+a_{0, l} \\
& P_{\text {loss }, 2 / 4}=a_{2, r} \tau_{w, 2 / 4}^{2}+a_{1, r} \tau_{w, 2 / 4}+a_{0, r}
\end{aligned}
$$


The total power loss as predicted by the quadratic function is

$$
\begin{gathered}
P_{l o s s}=a_{2, l}\left(\tau_{w, 1}^{2}+\tau_{w, 3}^{2}\right)+a_{2, r}\left(\tau_{w, 2}^{2}+\tau_{w, 4}^{2}\right) \\
+a_{1, l}\left(\tau_{w, 1}+\tau_{w, 3}\right)+a_{1, r}\left(\tau_{w, 2}+\tau_{w, 4}\right)+a_{0, l}+a_{0, r}
\end{gathered}
$$

and substituting for $\tau_{w, 1 / 3}$ and $\tau_{w, 2 / 4}$ using equation (11):

$$
\begin{gathered}
P_{\text {loss }}=a_{2, l}\left(\left[\tau_{w, l}^{\text {even }}-\Delta \tau_{w, l}\right]^{2}+\left[\tau_{w, l}^{\text {even }}+\Delta \tau_{w, l}\right]^{2}\right)+ \\
a_{2, r}\left(\left[\tau_{w, r}^{\text {even }}-\Delta \tau_{w, r}\right]^{2}+\left[\tau_{w, r}^{\text {even }}-\Delta \tau_{w, r}\right]^{2}\right)+a_{1, l}\left(\left[\tau_{w, l}^{\text {even }}-\right.\right. \\
\left.\left.\Delta \tau_{w, l}\right]+\left[\tau_{w, l}^{\text {even }}+\Delta \tau_{w, l}\right]\right)+a_{1, r}\left(\left[\tau_{w, r}^{\text {even }}-\Delta \tau_{w, r}\right]+\right. \\
\left.\left[\tau_{w, r}^{\text {even }}-\Delta \tau_{w, r}\right]\right)+2 a_{0, l}+2 a_{0, r} .
\end{gathered}
$$

The total power loss can thus be written as

$$
P_{\text {loss }}=2 a_{2, l} \Delta \tau_{w, l}^{2}+2 a_{2, r} \Delta \tau_{w, r}^{2}+\text { const. }
$$

The solution which minimizes the total power loss is thus given by $\Delta \tau_{w, l}=\Delta \tau_{w, r}=0$, i.e. $\boldsymbol{\tau}_{w}=\boldsymbol{\tau}_{w}^{e v e n}$. This result implies that when the same motor type is used at each of the four wheels, the potential for motor power loss minimization using quadratic programming is limited; more complex functions are required to approximate the variation in power loss across the torque-speed space. The solutions obtained for the optimal wheel torque by the direct quadratic and look-up table-based motor power loss cost functions are contrasted in a simulation approach, described in the next section.

\section{Simulation Study}

\section{1) Vehicle Properties}

Simulations are performed with a four wheel drive fully electric vehicle fitted with four individually-controlled onboard motors. The mass of the vehicle is $1963 \mathrm{~kg}$ and the half-track is $0.81 \mathrm{~m}$. The front and rear semi-wheelbases are $1.1 \mathrm{~m}$ and $1.6 \mathrm{~m}$, respectively, the transmission ratio is fixed at $10: 1$ and the wheel radius is $0.36 \mathrm{~m}$.

\section{2) Maneuvers}

Three maneuvers are simulated in this investigation. The first two consist of straight-ahead driving at constant accelerations of $1 \mathrm{~m} / \mathrm{s}^{2}$ and $2 \mathrm{~m} / \mathrm{s}^{2}$ for $60 \mathrm{~s}$, each from an initial speed of $50 \mathrm{~km} / \mathrm{h}$. The final maneuver is a ramp steer with a steering wheel gradient of $10 \%$ at a constant acceleration of $1 \mathrm{~m} / \mathrm{s}^{2}$ and an initial velocity of $50 \mathrm{~km} / \mathrm{h}$; the peak steering wheel angle is reached after $8 \mathrm{~s}$ in the ramp maneuver.

\section{3) Electric Motor Types and Characteristics}

Three different types of electric motor (with corresponding motor maps for each) are considered, which are: a switched reluctance (SR) motor, a permanent magnet (PM) motor, and a brushless direct current (BLDC) motor. Data concerning the the efficiency at different operating points for the SR and PM motors are obtained from experimental measurements of the respective motor manufacturers. The BLDC motor map is based on the curves provided by Chen and Wang [6,7].

The motor efficiency maps, shown in Figure 3, are scaled according to a set relationship regarding the maximum and minimum torques that can be produced at different motor speeds. The shapes of the power loss characteristic in the torque-speed range are distinct for the three motor types. In particular, the BLDC motor has a more pronounced variation in efficiency at different levels of torque than the other motor types.

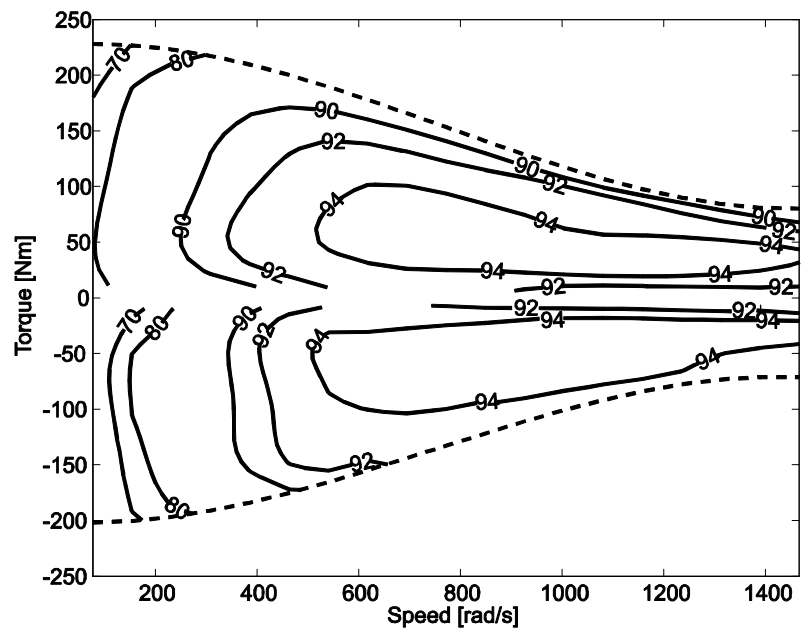

(a) Switched reluctance motor

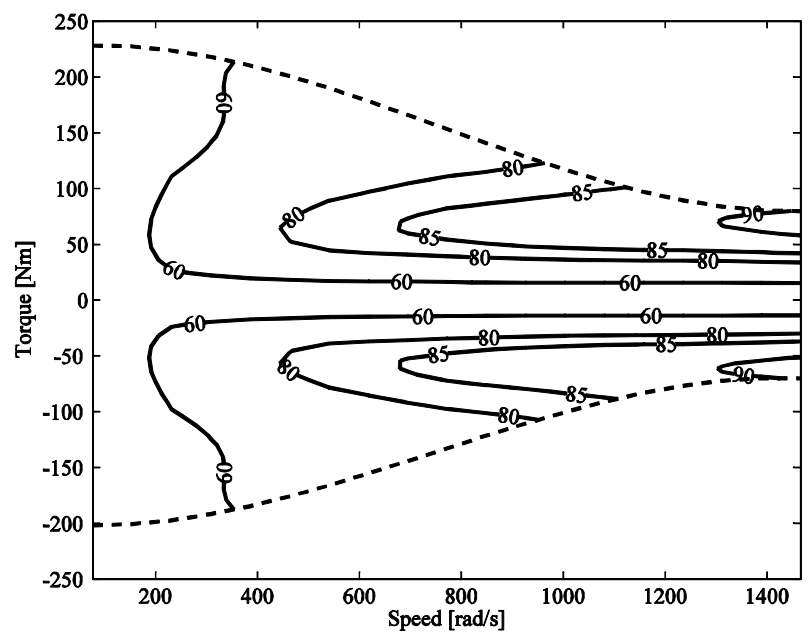

(b) Permanent magnet motor

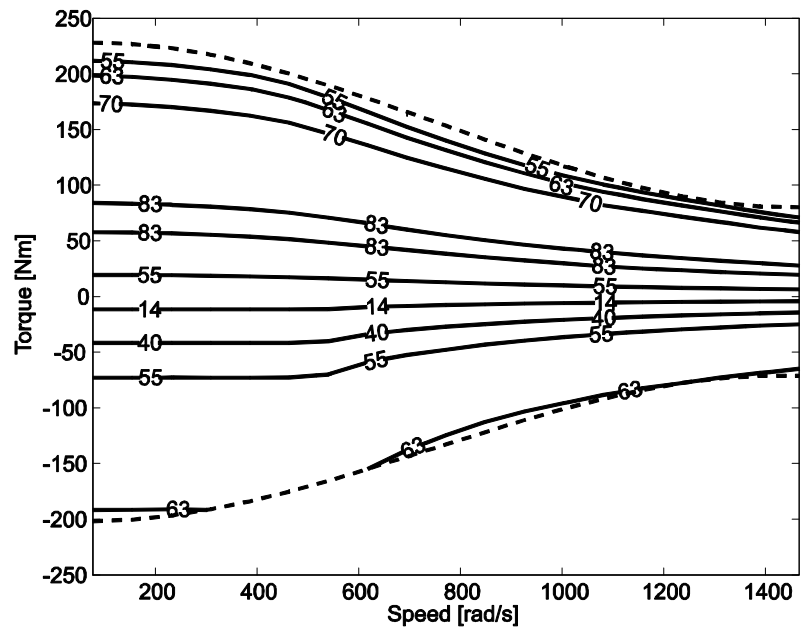

(c) Brushless direct current motor [6,7]

Figure 3. Efficiency characteristics for three different motor types, with dashed lines indicating minimum and maximum torques. 


\section{4) Simulation}

Solutions for the wheel torques at each time point according to the various control allocation methods during the different maneuvers and for different types of motor are generated in MATLAB. Data corresponding to the motor speed vector, $\boldsymbol{\omega}_{m}$, and also the virtual control, $\boldsymbol{v}$ (containing the desired traction force and yaw moment targets), are produced using a vehicle model constructed with IPG CarMaker. This model incorporates: i) a detailed powertrain model that allows simulation of the drivetrain dynamics, and ii) the control structure in Figure 1 with the evenly distributed control allocation providing $\boldsymbol{\tau}_{w}^{e v e n}$. Values of $\boldsymbol{\omega}_{m}$ and $\boldsymbol{v}$ during the three types of maneuver are thus produced via the IPG CarMaker model and subsequently used in the offline MATLAB-based simulation.

\section{5) Data Analysis}

For each simulated maneuver, the mean power loss, $\bar{P}_{\text {loss }}$, is computed as

$$
\bar{P}_{\text {loss }}=\frac{1}{T} \int_{0}^{T} P_{\text {loss }}(t) d t .
$$

Furthermore, equation (18) is used to determine the deviation of the optimization based solution, $\boldsymbol{\tau}_{w}^{o p t i}$, from the control allocation method, providing $\boldsymbol{\tau}_{w}^{\text {even, }}$, in which the traction torques and yaw moments are distributed evenly between the front and rear axles.

$$
\bar{\tau}_{w}^{\text {diff }}=\frac{1}{T} \int_{0}^{T} \sqrt{\frac{1}{4} \sum_{i=0}^{4}\left(\tau_{w, i}^{o p t i}(t)-\tau_{w, i}^{\text {even }}(t)\right)^{2}} d t
$$

This measure is the mean (with respect to time) of the root mean square (with respect to the four wheels) difference between the solution based on optimization (section B.3) and that based on equal traction torque and yaw moment on the front and rear axles (section B.2).

\section{RESULTS AND DISCUSSION}

Table I shows the normalized root mean square errors of the quadratic approximation of the motor power data for the three motor types. On average, the quadratic function represents the motor loss at different speeds and torques with good accuracy.

TABLE I. NORMALIZED ROOT MEAN SQUARE ERRORS (NRMSE) OF QUADRATIC FIT TO EXPERIMENTAL DATA FOR THREE MOTOR TYPES

\begin{tabular}{|c|c|}
\hline Motor type & NRMSE [\%] \\
\hline SR & 4.91 \\
\hline PM & 2.07 \\
\hline BLDC & 5.06 \\
\hline
\end{tabular}

The power losses incurred during straight-ahead driving at a constant acceleration of $1 \mathrm{~m} / \mathrm{s}^{2}$ computed with the even torque allocation method and the optimization scheme using the quadratic cost function and power loss look-up tables are shown in Table II.
TABLE II. MEAN POWER LOSSES AND DIFFERENCES IN WHEEL TORQUES DURING STRAIGHT-AHEAD DRIVING AT A CONSTANT ACCELERATION OF $1 \mathrm{M} / \mathrm{s}^{2}$

\begin{tabular}{|c|c|c|c|c|c|}
\hline \multirow{2}{*}{$\begin{array}{c}\text { Motor } \\
\text { type }\end{array}$} & $\begin{array}{c}\bar{P}_{\text {loss }}^{\text {even }} \\
{[\mathrm{kW}]}\end{array}$ & $\begin{array}{c}|c| \\
\bar{P}_{\text {loss }}^{\text {opti }} \\
{[\mathrm{kW}]}\end{array}$ & $\begin{array}{c}\bar{\tau}_{w}^{\text {diff }} \\
{[\mathrm{Nm}]}\end{array}$ & $\begin{array}{c}\bar{P}_{\text {loss }}^{\text {opti }} \\
{[\mathrm{kW}]}\end{array}$ & $\begin{array}{c}\bar{\tau}_{w}^{\text {diff }} \\
{[\mathrm{Nm}]}\end{array}$ \\
\hline $\mathrm{SR}$ & 4.64 & 4.64 & $<1$ & 4.17 & 253 \\
\hline $\mathrm{PM}$ & 16.0 & 16.0 & $<1$ & 16.0 & 14.3 \\
\hline BLDC & 24.9 & 24.9 & $<1$ & 24.2 & 151 \\
\hline
\end{tabular}

Significant power loss reductions are achieved during the 1 $\mathrm{m} / \mathrm{s}^{2}$ acceleration maneuver for the switched reluctance motor, but only when the look-up table data is used as the cost function. For each motor type, the optimization based on minimizing a quadratic function representative of power loss produces virtually identical results to the simpler control allocation method in which the wheel torques are evenly distributed.

The results for the higher acceleration of $2 \mathrm{~m} / \mathrm{s}^{2}$ are shown in TABLE III. The mean operating point for this maneuver is different from the lower acceleration simulation, having a far higher torque demand. At this operating point, the motors are more efficient, and significant reductions in power loss using the optimization scheme could not be made.

TABLE III. MEAN POWER LOSSES AND DIFFERENCES IN WHEEL TORQUES DURING STRAIGHT-AHEAD DRIVING AT A CONSTANT ACCELERATION OF $2 \mathrm{M} / \mathrm{s}^{2}$

\begin{tabular}{|c|c|c|c|c|c|}
\hline \multirow{2}{*}{$\begin{array}{c}\text { Motor } \\
\text { type }\end{array}$} & $\begin{array}{c}\bar{P}_{\text {loss }}^{\text {even }} \\
{[\mathrm{kW}]}\end{array}$ & $\begin{array}{c}|c| \\
\bar{P}_{\text {loss }}^{\text {opti }} \\
{[\mathrm{kW}]}\end{array}$ & $\begin{array}{c}\bar{\tau}_{w}^{\text {diff }} \\
{[\mathrm{Nm}]}\end{array}$ & $\begin{array}{c}\bar{P}_{\text {loss }}^{\text {opti }} \\
{[\mathrm{kW}]}\end{array}$ & $\begin{array}{c}\bar{\tau}_{w}^{\text {diff }} \\
{[\mathrm{Nm}]}\end{array}$ \\
\hline $\mathrm{SR}$ & 11.8 & 11.8 & $<1$ & 11.8 & 6.94 \\
\hline $\mathrm{PM}$ & 38.2 & 38.2 & $<1$ & 38.1 & 13.0 \\
\hline BLDC & 52.5 & 52.5 & $<1$ & 52.4 & 32.5 \\
\hline
\end{tabular}

For the ramp steer maneuver, the results (TABLE IV) show that power savings for the switched reluctance and brushless direct current motors could be achieved using the optimization incorporating the motor loss look-up table (based on the efficiency map data).

TABLE IV. MEAN POWER LOSSES AND DIFFERENCES IN WHEEL TORQUES DURING RAMP STEER MANEUVER AT A CONSTANT ACCELERATION OF $1 \mathrm{M} / \mathrm{s}^{2}$

\begin{tabular}{|c|c|c|c|c|c|}
\hline \multirow{2}{*}{$\begin{array}{c}\text { Motor } \\
\text { type }\end{array}$} & $\begin{array}{c}P_{\text {loss }}^{\text {even }} \\
{[\mathrm{kW}]}\end{array}$ & $\begin{array}{c}|c| \\
P_{l o s s}^{\text {opti }} \\
{[\mathrm{kW}]}\end{array}$ & $\begin{array}{c}\bar{\tau}_{w}^{\text {diff }} \\
{[\mathrm{Nm}]}\end{array}$ & $\begin{array}{c}P_{\text {loss }}^{\text {opti }} \\
{[\mathrm{kW}]}\end{array}$ & $\begin{array}{c}\bar{\tau}_{w}^{\text {diff }} \\
{[\mathrm{Nm}]}\end{array}$ \\
\hline SR & 4.06 & 4.06 & $<1$ & 3.80 & 205 \\
\hline $\mathrm{PM}$ & 15.2 & 15.2 & $<1$ & 15.2 & 7.06 \\
\hline BLDC & 22.4 & 22.4 & $<1$ & 18.1 & 174 \\
\hline
\end{tabular}


However, wheel torques allocated according to a quadratic programming approach produced very similar wheel torques to the even wheel torque allocation scheme.

In summary, the simulation results show that motor power loss reductions can be achieved through an optimization approach seeking to simultaneously the realize traction force and yaw rate targets and minimize the total motor power loss, based on experimentally-derived power loss look-up tables. Whether or not significant savings can be made depends on both the maneuver, which sets the region of the efficiency maps in which the motors operate, and the motor type. Due to the shape of the efficiency map, significant power loss reductions (compared to evenly distributed control allocation) could not be made for the permanent magnet motor type for the specific maneuvers simulated.

Due to the symmetry of the function and also the fact that the front and rear wheels on each side of the vehicle are approximately equal, a quadratic cost function for representing the motor power losses is not an effective approach to minimizing motor power losses when the same motor type is used at each of the four wheels. In addition to consistently generating a solution that is virtually identical to the simpler even-distribution wheel torque allocation method, the quadratic has the disadvantage of reduced accuracy in the representation of the power losses (as predicted by the motor maps) than more complex functions. Nevertheless, quadratic functions are appealing since efficient algorithms for their solution exist which can be applied in real time, and also because, under certain conditions, there will be a unique global minimum. The results of this study indicate that more complex approximations are needed for this optimization application; such approximations, in addition to being more computationally demanding to solve, may also have multiple local minima, further increasing the challenge of their application online.

There are several simplifications used in this study. Simulated data from a more complex model have been used for the motor speeds and virtual control signal for each maneuver during the offline simulation. Effects of the different wheel torque allocation methods on the motor speeds and virtual control are thus not included. The effects of the control allocation scheme on the wheel and motor speeds are minor and will not substantially affect the operating points of the motors and thus the motor efficiency during the simulation.

In this study, the energy losses from the motor units have been considered. It should be noted that there are multiple sources of energy loss during vehicle operation, and several of these can be strongly influenced by control allocation. For example, energy losses occur due to longitudinal and lateral tire slip, and both of these are influenced by the distribution of wheel torques applied to realize the traction force and yaw moment targets. In order to truly minimize energy consumption, these additional sources of energy loss should be considered during future wheel torque allocation development.

\section{CONCLUSIONS}

The feasibility of using optimization to reduce power losses from the electric motor drives during various maneuvers of a fully electric vehicle with individually-controllable electric motors has been explored. Using an optimization scheme incorporating a look-up table of motor loss data derived from the efficiency maps, significant power loss reductions are achieved for two types of electric motor. Both the simplified analysis and simulation results of this study show that when the same type of motor is used at each wheel, optimization based on a quadratic cost function representing the motor power losses does not yield improvements over a simpler scheme in which the wheel torque contributions for traction force and yaw moment are realized equally from the wheels of the front and rear axles. More complex functions are thus needed as cost functions for motor power loss minimization.

\section{ACKNOWLEDGMENT}

The research leading to these results has received funding from the European Union Seventh Framework Programme FP7/2007-2013 under grant agreement no. 284708.

\section{REFERENCES}

[1] A. Van Zanten, R. Ehardt and G. Pfaff, "VDC, the vehicle dynamics control system of Bosch", SAE Paper 950759, 1995.

[2] L. De Novellis, A. Sorniotti, P. Gruber, L. Shead, V. Ivanov and K. Hoepping, "Torque vectoring for electric vehicles with individually controlled motors: state-of-the-art and future developments" $26^{\text {th }}$ International Electric Vehicle Symposium (EVS26), 2012.

[3] M. Naraghi, A. Roshanbin and A. Tavasoli, "Vehicle stability enhancement and adaptive optimal approach to the distribution of tyre forces", Proceedings of the Instituion of Mechanical Engineers, Part D: Journal of Automotive Engineering, vol. 224, pp. 443-453, 2010.

[4] D. Li and X. Yu, "Optimal tyre force distribution in integrated chassis control", in $21^{\text {st }}$ International Symposium on Dynamics of Vehicles on Road and Tracks, 2009.

[5] J. Wang and R. Longoria, "Coordinated vehicle dynamics control with control distrubution," in American Control Conference, 2006.

[6] Y. Chen and J. Wang, "Energy-efficient control allocation with applications on planar motion control of electric ground vehicles", in 2011 Americal Control Conference.

[7] Y. Chen and J. Wang, "Fast and global optimal energy-efficient control allocation with applications to over-actuated electric ground vehicles," IEEE Transactions on Control Systems Technology, 2012, vol. 20, pp. 1202-1211

[8] A. Sorniotti, T. Holdstock, M. Everitt, M. Fracchia, F. Viotto, C. Cavallino and S. Bertolotto, "A novel clutchless multiple-speed transmission for electric axles", International Journal of Powertrains, 2013, vol. 2, no. 2/3, pp.103-131.

[9] J.H. Plumlee, D.M. Bevly and A.S. Hodel, "Control of a ground vehicle using quadratic programming based on control allocation techniques," Proceedings of the 2004 American Control Conference, pp. 4704-4709.

[10] K. Koibuchi, "Behavior control system of vehicle distinctive of oversteered and understeered conditions," 1997, US Patent 5,702,165.

[11] L. De Novellis, A. Sorniotti and P. Gruber, "Optimal wheel torque distribution for a four-wheel-drive fully electric vehicle," SAE International Journal of Passenger Cars, 2013, 6(1), pp 128-136. 